\title{
Novel Hybrid Cooling Concept for Battery Thermal Management Design
}

\author{
Yuyang Wei, Martin Agelin-Chaab \\ Automotive Center of Excellence, Faculty of Engineering and Applied Science \\ University of Ontario Institute of Technology \\ 2000 Simcoe Street North, Oshawa, Ontario, L1H 7K4, Canada \\ Yuyang.wei@uoit.ca; Martin.agelin-chaab@uoit.ca
}

\begin{abstract}
Electric vehicles (EVs) is one of the most promising solutions to global warming and fossil fuel crisis. The key to EV development is the battery. Li-ion battery has been the most popular choice in the industry for a variety of advantages over the others. However, battery performance is heavily related to its working temperature, and the health of battery a pack in long-term is hugely affected by temperature uniformity. The optimal working temperature ranges from $25^{\circ} \mathrm{C}$ to $40^{\circ} \mathrm{C}$, while the temperature non-uniformity should be lower than $5^{\circ} \mathrm{C}$. A novel hybrid cooling concept for battery applications is proposed and experimentally proved in this study. The concept utilizes any combination of conductive, convective, and evaporative phase change cooling effects. The concept takes nearly no extra power from a normal air cooling method by using capillary effect as the driving force of water coolant, but achieves a higher cooling efficiency and better temperature uniformity. The concept may recycle $\mathrm{A} / \mathrm{C}$ condensate so the water coolant could be instantly consumed at its generation and hence adds no extra weight to the vehicle. The air and water coolant after use will be released to the ambient without harming the environment. The results show a higher cooling efficiency and better temperature uniformity over the pack. The concept was able to retain both of the maximum working temperature and non-uniformity under the limits.
\end{abstract}

Keywords: Electric Vehicle, Lithium-Ion Battery, Thermal Management System, Hybrid Cooling.

\section{Introduction}

Electric vehicle (EV) has been in an increasing demand within the past decade. Two types of EV are mostly found in the market: hybrid (HEV) and full-electric plug-in electric vehicle (PEV). No matter which type the EV is, battery technology is the key to development and market share of EV. Lithium-ion battery (LIB) is so far the best choice for EV energy unit design because of its high energy density, long cycle life, low self-discharge rate, and great efficiency compared to the others [1]. Meanwhile, performance and health of a battery are heavily related to its working temperature despite whatever battery chemistry. Hence, battery thermal management is critical to EV battery applications. The optimal working temperature for common LIBs is reported between $25^{\circ} \mathrm{C}$ to $40^{\circ} \mathrm{C}[2,3]$, and the temperature nonuniformity over the pack, calculated as the difference between the highest and the lowest cell temperature, should not exceed $5^{\circ} \mathrm{C}$, however, the actual working temperature and non-uniformity can be much higher in cases of high current draining and thermal runaway. The charge and discharge current load on a rechargeable battery cell is measured as $\mathrm{C}$-rate. $1 \mathrm{C}$ stands for a current rate reading that is same as the magnitude of the battery nominal capacity. A high C-rate in both charge and discharge increases the load on the battery and hence the heat generation. For EVs, the situations such as accelerating and high load on air-conditioning significantly increase C-rate. This increase the thermal instability and hence battery degradation or even safety issues. Therefore, battery thermal management is critical for EV applications. Three cooling methods are widely studied in the field of EV battery thermal management: (1) air-cooling, (2) liquid cooling, and (3) phase-change material (PCM).

Air-cooling is the most traditional cooling method in industries. It comes with simple and light-weight structure, low running power, low development and maintenance cost, and environmental friendliness. Hence, it is popular although the low thermal conductivity and heat capacity of air lower its cooling efficiency. Additionally, the air-cooling efficiency is highly dependent on the ambient temperature. Prediction of the airflow behaviors within a battery pack can be difficult. For example, a pack of cylindrical batteries cooled by a unidirectional airflow was reported with a higher cooling efficiency by using in-line layout than the staggered layout [1], while some studies made opposite statement [4]. So the design criteria for the air-cooling systems for different battery sizes and pack dimensions may not be consistent. Other designs are similar to each other by having converging inlet and diverging outlet located at the top and bottom of the 
battery pack or modules, to make the air pressure uniform over the energy unit [5-8]. However, their packs are geometrically asymmetric by the converging inlet and diverging outlet duct designs, so they may be hard to assemble in EVs as battery modules. Therefore, reciprocating flows are studied for uniformity improvement and showed that the uniformity is positively correlated to the frequency of flow reciprocation [4, 9-12]. Meanwhile, the high frequencies of reciprocating flow are expensive to implement and will decrease system reliability dramatically.

Liquids with much higher thermal conductivity and heat capacity than air are generally more effective in cooling. However, normal liquid-cooling systems are complicated in structure and system design, heavyweight, costly and higher running power, because of requirements of a recirculating system including pumping, piping and flowrate control. Some existing liquid cooling methods employ glycol or water as the coolant, however, different coolant materials could also be used. Pouch or prismatic cells were better studied than the cylindrical cells with liquid cooling since they allow simpler and compact design of cooling plates $[13,14]$. The coolant flow rate for normal EV battery pack can be as high as about $10 \mathrm{~L} / \mathrm{min}$ and hence the energy consumption by the pump can be high, resulting in a dramatic drop in EV driving range [5].

PCM cooling is a purely passive and requires no extra energy to work. They theoretically have the highest cooling efficiency by taking out latent heat. PCM cooling comes with the best uniformity since the heat source will be directly dipped into and fully covered by the coolant [15]. However, PCM can also be heavy and not environmentally friendly. Their material usually cost much more than those used in the other cooling methods. The heat taken out by PCM may also need to be extracted by other cooling methods because the total heat capacity of a certain mass of PCM is limited [16, 17]. Another PCM-like cooling approach is the heat pipe, which contains complex inner structures and special chemicals with low boiling points, making it too expensive for industrial applications.

For all these mentioned studies, it can be concluded that (1) the air-cooling method is usually simple in structure, light-weight, energy-saving, but has the worst cooling performance. Improvement in cooling performance could sacrifice the simplicity and the battery pack energy density; (2) the liquid cooling method performs better than air, but it needs a circulation systems which adds complexity, weight, and energy consumption; (3) the PCM method can provide the best cooling performance both in terms of the temperature rise and uniformity, however, since the thermal capacity is dependent on mass, PCM systems are usually heavy or require additional cooling systems to extract the heat from the PCM pack; and (4) coolants chosen for the liquid cooling and the PCM systems may not be environmentally friendly.

The above conclusions motivated a concept of hybrid EV battery cooling that combines at least two cooling methods directly working on the battery cell surface. The goal is to improve system simplicity, weight reduction, energy saving, and environmental friendliness.

\section{System Design}

In this paper, a hybrid thermal management method for electric vehicles (EVs) is proposed. The design is based on a simple air-cooling duct. A series of hydrophilic fiber channels, where the water coolant is driven through purely by natural capillary or gravity force, is exposed to the air flow to extract the latent heat from the battery. So the system can utilize enhanced water vaporization by air convection to achieve an effective cooling. Figure 1(a) demonstrates the proposed concept being tested in this study.

The proposed design is applicable to any type of battery (cylindrical, prismatic, or pouch type in different sizes or dimensions) or electric energy supply that requires cooling. The hydrophilic fiber strip can be either contacted or contactless to the battery surface, served as fiber channel for the conductive and evaporative water coolant. The air duct in this test is placed horizontally, another design variation can be made by multiple vertical air ducts. The capillary effect can not only be the coolant driving force but also constrain the water from leaking out from the fiber.

Figure 1(b) shows the schematic overview of one possible system configuration. Different cooling stages can be designed, for example, a pure air-cooling mode can be assigned as first-stage cooling intended for the small-load driving mode, a second-stage water-cooling is suitable for a medium-load driving mode, and a final-stage hybrid cooling mode is able to cool down the batteries in extreme-load driving condition. Condensate from the air-conditioner (A/C) can be recycled as the water coolant as well. A low-power pump might be required only for transporting the condensate to the reservoir, while the coolant in the cooling system is driven purely by natural forces. No extra power is required from the batteries. The air and the water coolant can be released to the ambient after use without harming the environment. 
(a)

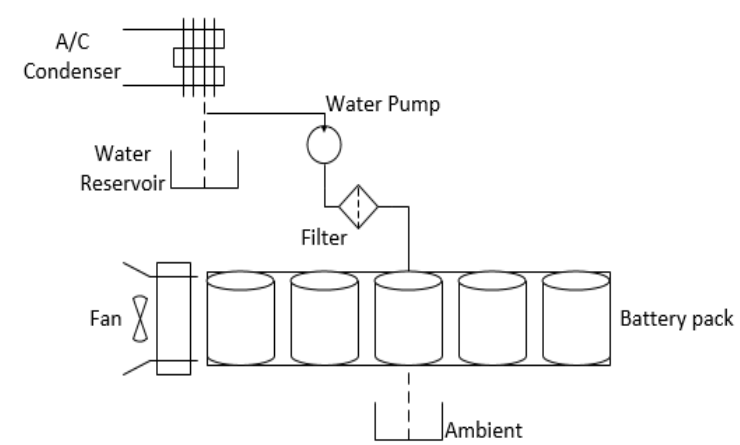

(b)

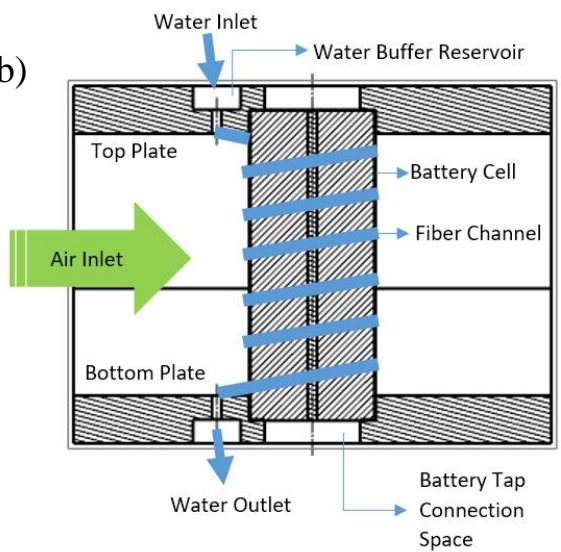

Fig. 1: Schematics of a conceptual system configuration of the proposed hybrid cooling system.

\section{Experiment}

\subsection{Measurement Equipment}

A single Li-ion battery (Efest IMR $266505200 \mathrm{mAh}$ ) was used in each set of experiments. The fiber channels were made from weaved fiber strips. A 4-channel battery charger (Reaktor Quadcore $4 \times 300 \mathrm{~W} 20 \mathrm{~A}$ ) and a multifunctional high-power discharger (TDI RBL488) were used. Temperatures were measured by both an infrared thermal camera (SPI IRXP-5000) and T-type thermocouples that was attached onto the battery surface at the location Ar1 shown in Figure 5. The thermocouples were connected to a data acquisition board (National Instrument cDAQ-9188XT), logged with LabView software. A multifunction anemometer (Proster TL107) was used to measure the inlet/outlet wind speed and ambient temperatures. A electronic weight scale (OHAUS PA153) was used to measure the water evaporation rate.

\subsection{Temperature Measurement}

Figure 2 shows the schematic of experimental setups. Four tests were designed to compare the cooling performance among air-cooling only, water-cooling only, and the hybrid cooling. The case of no cooling was made as the baseline. All the experiments were conducted at room temperature around $21^{\circ} \mathrm{C}$ to $23^{\circ} \mathrm{C}$. The inlet air velocity for the air-, the water-, and the hybrid cooling test was set to around $0.7 \mathrm{~m} / \mathrm{s}$ by the anemometer, which is consistent with the study by Yang [1]. For the water cooling and hybrid cooling tests, the water temperature was maintained at around $20^{\circ} \mathrm{C}$, and the fiber channel inlet water flow rate was determined to be about 0.015 to $0.020 \mathrm{~g} / \mathrm{s}$.

Seven spots of infrared temperature measurement were assigned to the back surface of the batteries as shown in Figure 3 for all the tests. The transverse temperature differences were measured at the 3 spots at the top ( $\mathrm{Sp} 1, \mathrm{Sp} 2$, and $\mathrm{Sp} 3)$ and the other 3 spots (Sp5, Sp6, and Sp7) at the bottom. The axial temperature differences were measured at Sp2, $\mathrm{Sp} 4$, and Sp6. An extra areal infrared temperature measurement was placed at Ar1 in Figure 5. It measured the average temperature at the thermocouple location.

For all the temperature measurements, the batteries were charged to $4.0 \mathrm{~V}$ using constant current and constant voltage $(\mathrm{CC}-\mathrm{CV})$ method with charging currents starting at $1.0 \mathrm{~A}(<0.2 \mathrm{C})$. The charging processes were cut off when the charging current dropped to $0.1 \mathrm{~A}(\cong 0.02 \mathrm{C})$. The discharge cut off voltage (under-voltage) limit was set as $2.5 \mathrm{~V}$. The batteries were discharged with a constant current $(\mathrm{CC})$ of $6.0 \mathrm{~A}(\cong 1.15$

(a)

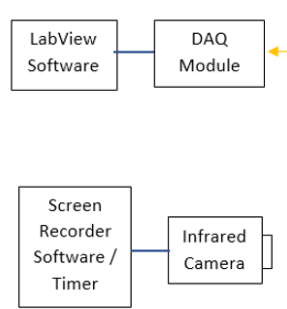

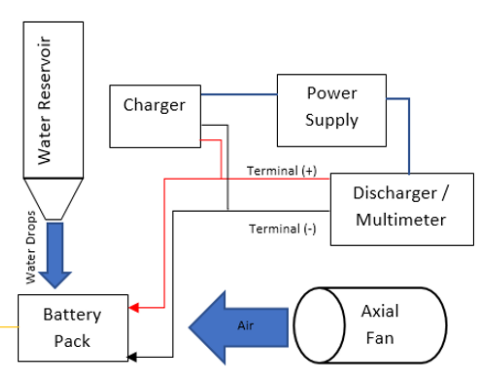

(b)

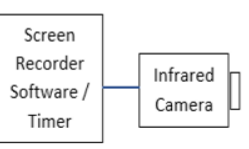

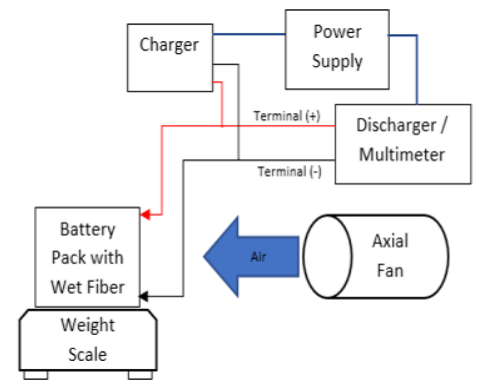

Fig. 2: Schematic of experimental setups of (a) temperature rise \& uniformity measurements, and (b) water evaporation measurement. 


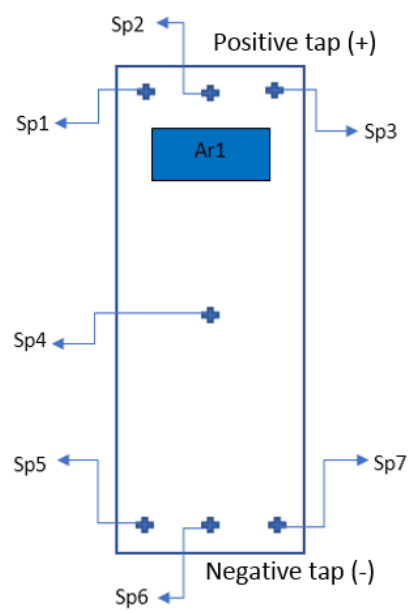

Fig. 3: Locations of thermal camera sensing spots $(\mathrm{Sp})$ and areas $(\mathrm{Ar})$ on the battery cell surface.

\subsection{Water Evaporation Rate Measurement}

In the evaporation measurement test, 10 samples were taken. The battery in the pack was winded with fiber channel saturated wetted but without any leaks. An initial pack weight was taken from the weight scale with the fan on, then started discharging. A final pack weight was recorded after the discharge was completed. The quantity of water evaporation was taken as the average difference between the initial and final weights.

\subsection{Error Analysis}

The uncertainty analysis was made according to Moffat [20]. The average total uncertainties for the infrared camera and the thermocouples could be calculated by the absolute bias given by the equipment specification ( $\pm 2 \%$ for the infrared camera and $\pm 0.5^{\circ} \mathrm{C}$ for the thermocouples), and the relative precision (or so-called relative standard deviation) taken during the test. The uncertainties hence were estimated as $4.9 \%$ and $1.5 \%$ for the camera and the thermocouples respectively.

\section{Results and Discussion}

\subsection{Cooling Effectiveness}

Figure 4 shows the plots of the surface temperature rise measured using the thermocouples under the different cooling methods described above. By using the hybrid cooling method, the maximum temperature in the cell level decreased by $82.9 \%$ compared to the no-cooling method. Additionally, it decreased by $69.6 \%$ and $57.3 \%$ compared to the air- and water-cooling, respectively.

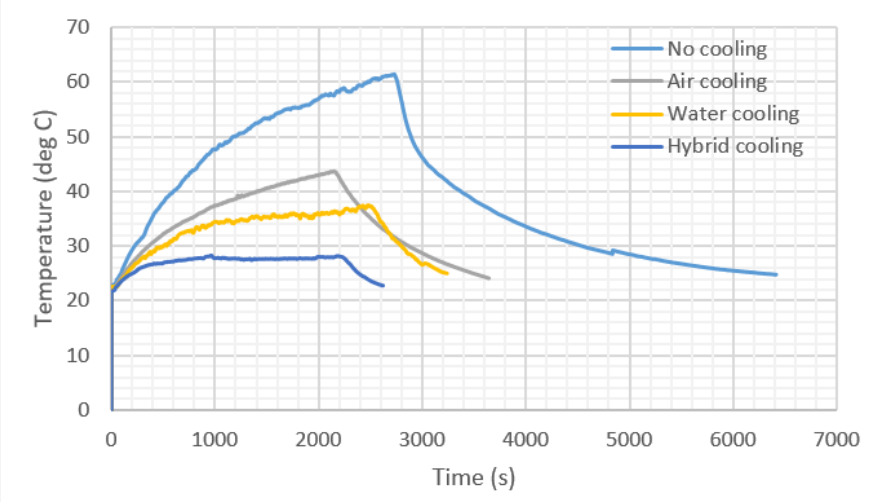

Fig. 4: Surface temperature change with time during a $1.15 \mathrm{C}$ constant current discharge followed by a constant current and constant voltage charge starting with $<0.2 C$ load.

\subsection{Temperature Uniformity}

As the transverse temperature differences are negligible, the temperature non-uniformity can be estimated as the temperature differences $\Delta \mathrm{T}$ between the $\mathrm{Sp} 2$ and $\mathrm{Sp} 6$ (i.e., $T_{S p 2}-T_{S p 6}$ ) during discharge. Figure 5, Figure 6, Figure 7, and Figure 8 show the temperature contours and the plot of local temperature difference $(\Delta \mathrm{T})$ versus discharge time for the no-cooling, air cooling, water cooling, and hybrid cooling, respectively. 
The uniformity was significantly improved by applying the proposed method. The maximum non-uniformity in the hybrid-cooling test was reported as $4^{\circ} \mathrm{C}$, and it was also the only case that could maintain the maximum temperature difference stable under $5^{\circ} \mathrm{C}$ over the whole surface.

(a)

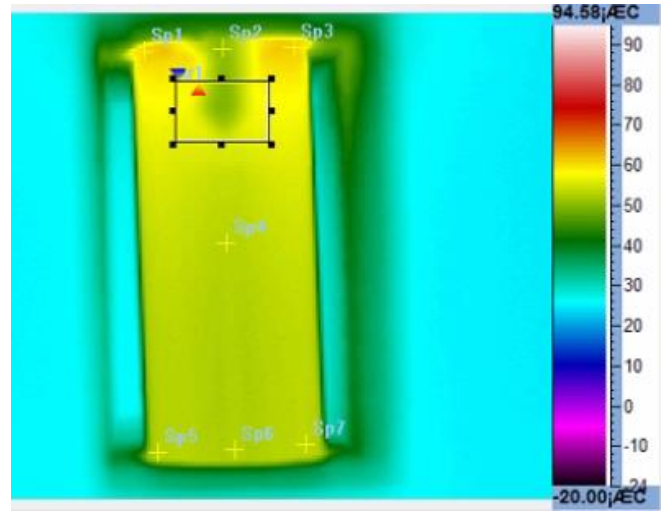

(b)

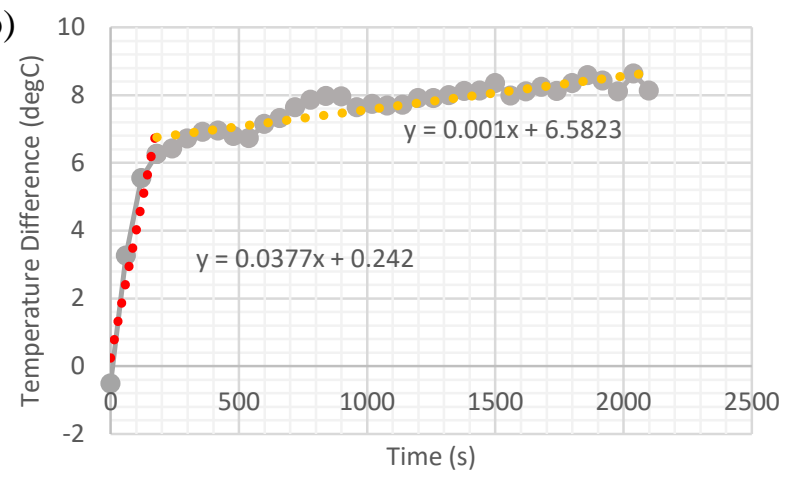

Fig. 5: (a) Temperature contour obtained by the thermal camera at 2100s of discharging, and (b) temperature non-uniformity change over discharging time for the no-cooling test.

(a)

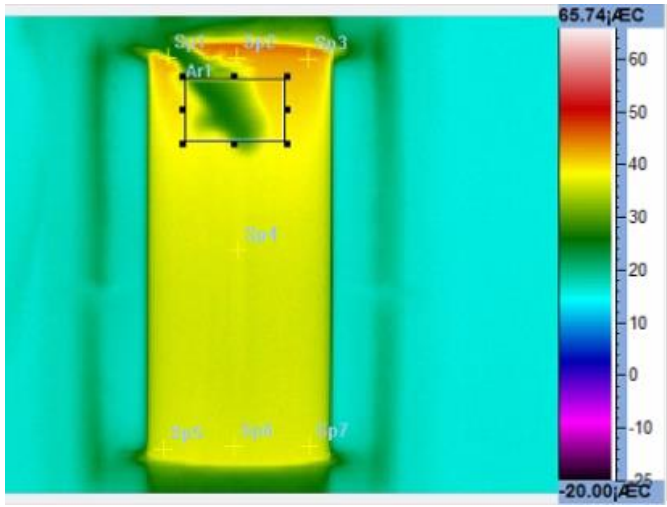

(b)

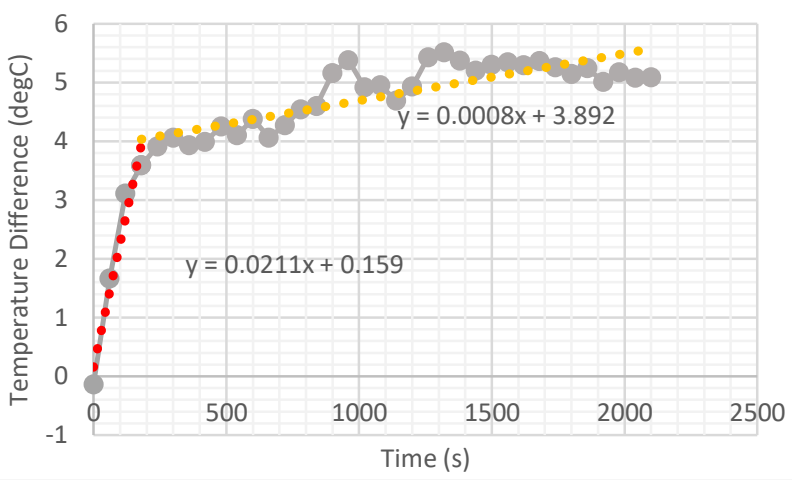

Fig. 6: (a) Temperature contour obtained by the thermal camera at 2100s of discharging, and (b) temperature non-uniformity change over discharging time for the air-cooling test.

(a)

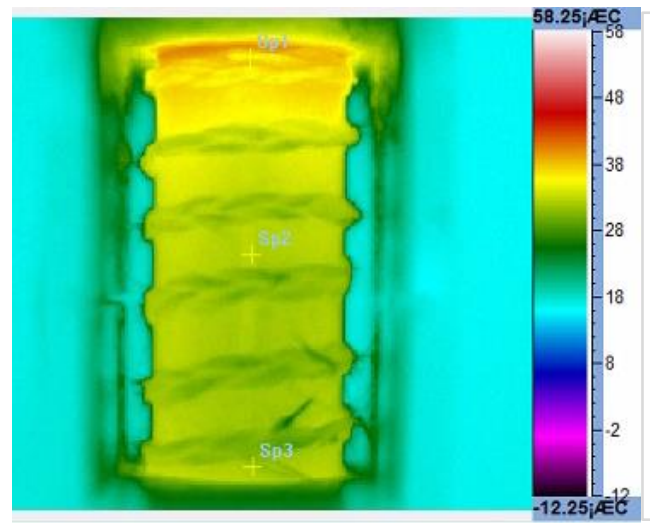

(b)

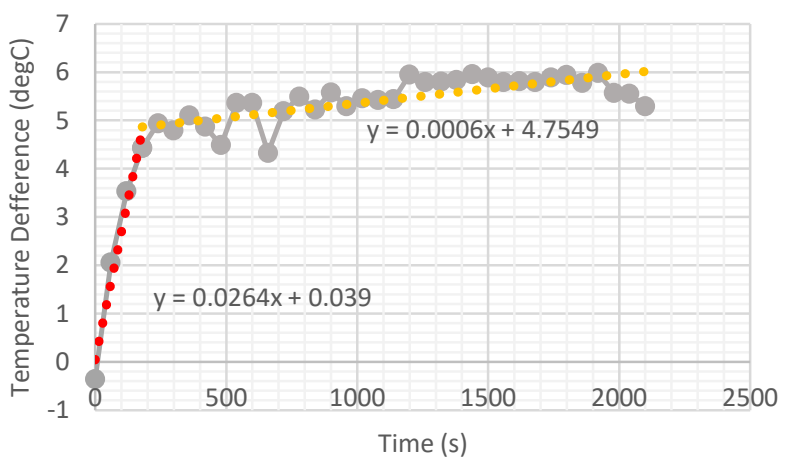

Fig. 7: (a) Temperature contour obtained by the thermal camera at 2100s of discharging, and (b) temperature non-uniformity change over discharging time for the water-cooling test. 
(a)

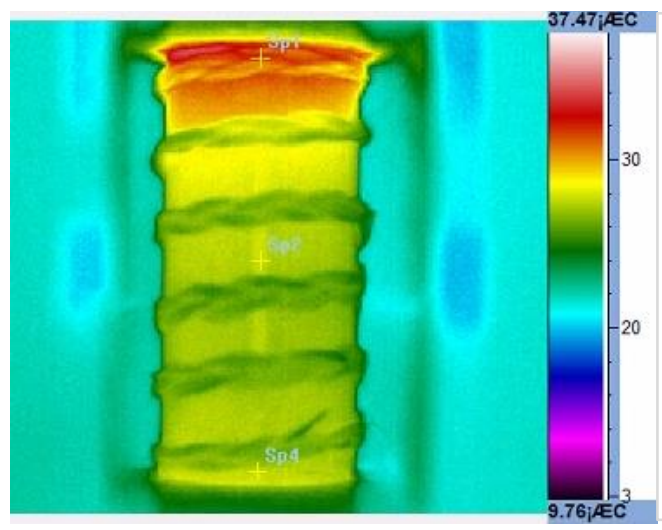

(b)

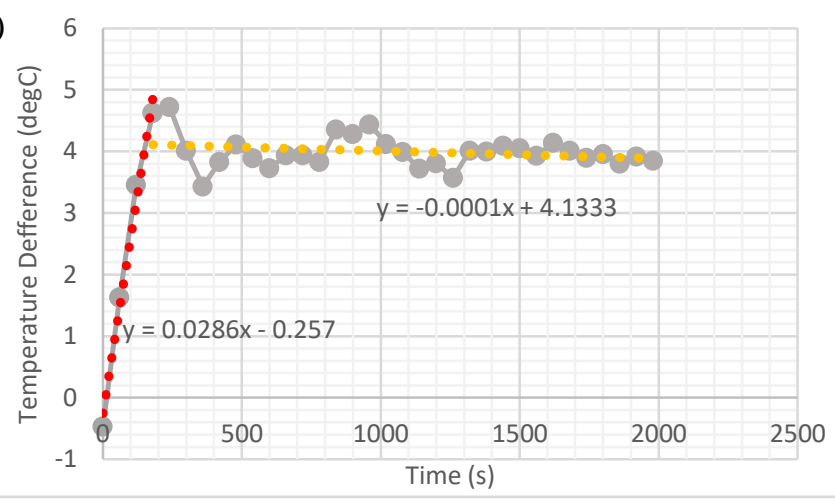

Fig. 8: (a) Temperature contour obtained by the thermal camera at 2100s of discharging, and (b) temperature non-uniformity change over discharging time for the hybrid cooling test.

\subsection{Evaporation}

The weight of the battery pack with wet fiber channels was measured as 115.185 grams at $t=0$ second. The water was dried out at $\mathrm{t}=1790$ second and the weight was measured as 114.000 grams. Thus, the evaporative mass flow rate can be averaged as $6.62 \times 10^{-4} \mathrm{~g} / \mathrm{s}$. As the latent heat taken by water evaporation $H$ was known as about $2260 \mathrm{~J} / \mathrm{g}$, the water phase change cooling power can be averaged to 1.50 watts. In addition, the heat generation rates for a single battery cell are usually lower than 5 watts $[18,19]$, thus the latent heat taken by the water evaporation can remove about $30 \%$ of the heat.

\section{Conclusion}

A novel hybrid cooling concept for battery applications is proposed and experimentally proved in this study. The concept utilizes any combination of conductive, convective, evaporative and phase change cooling effects. The results show a higher cooling efficiency and better temperature uniformity over the pack. By using the hybrid cooling method, the maximum temperature in the cell level decreased by $82.9 \%$ compared to the no-cooling method. Additionally, it decreased by $69.6 \%$ and $57.3 \%$ compared to the air- and water-cooling, respectively. The hybrid cooling test was reported as the only case with maximum non-uniformity below $5^{\circ} \mathrm{C}$. It must be noted that these results were achieved under the above experiments are the crudest, simplest and unidirectional airflow cooling with inline battery layout. Therefore, there is a great potential for improvement for the proposed hybrid cooling concept with further optimization.

The concept takes nearly no extra power from a normal air cooling method by using capillary effect as the driving force of water coolant, but achieve a higher cooling efficiency and better temperature uniformity. The concept may recycle $\mathrm{A} / \mathrm{C}$ condensate so the water coolant could be instantly consumed as it is generated and hence adds no extra weight to the vehicle. The air and water coolant after use will be released to the ambient environment without harming the environment since no chemical is used.

\section{Reference}

[1] Yang, N., Zhang, X., Li, G. and Hua, D. (2015), "Assessment of the forced air-cooling performance for cylindrical lithium-ion battery packs: A comparative analysis between aligned and staggered cell arrangements", Applied Thermal Engineering, 80, pp. 55-65.

[2] Rao, Z. and Wang, S. (2011), "A review of power battery thermal energy management", Renewable and Sustainable Energy Reviews, 15(9), pp. 4554-4571.

[3] Jaguemont, J., Boulon, L. and Dubé, Y. (2016), "A comprehensive review of lithium-ion batteries used in hybrid and electric vehicles at cold temperatures", Applied Energy, 164, pp.99-114.

[4] Tong, W., Somasundaram, K., Birgersson, E., Mujumdar, A.S. and Yap, C. (2016), "Thermo-electrochemical model for forced convection air cooling of a lithium-ion battery module", Applied Thermal Engineering, 99, pp. 672-682.

[5] Park, H. (2013), "A design of air flow configuration for cooling lithium-ion battery in hybrid electric vehicles", Journal of Power Sources, 239, pp. 30-36.

[6] Saw, L.H., Ye, Y., Tay, A.A.O., Chong, W.T., Kuan, S.H. and Yew, M.C. (2016), "Computational fluid dynamic and thermal analysis of lithium-ion battery pack with air cooling", Applied Energy, 177, pp. 783-792.

[7] Sun, H. and Dixon, R. (2014), "Development of cooling strategy for an air-cooled lithium-ion battery pack", Journal of Power Sources, 272, pp. 404-414. 
[8] Liu, Z., Wang, Y. and Zhang, J. (2014), "Shortcut computation for the thermal management of a large air-cooled battery pack", Applied Thermal Engineering, 66(1-2), pp. 445-452.

[9] Ling, Z., Wang, F., Fang, X., Gao, X. and Zhang, Z. (2015), "A hybrid thermal management system for lithium-ion batteries combining phase change materials with forced-air cooling", Applied Energy, 148, pp. 403-409.

[10] Fan, L., Khodadadi, J.M. and Pesaran, A.A. (2013), "A parametric study on thermal management of an air-cooled lithium-ion battery module for plug-in hybrid electric vehicles", Journal of Power Sources, 238, pp. 301-312.

[11] Sasmito, A., Birgersson, E., and Mujumdar, A. (2012), "A novel flow reversal concept for improved thermal management in polymer electrolyte fuel cell stacks", International Journal of Thermal Sciences, 54, pp.242-252.

[12] Mahamud, R. and Park, C. (2011), "Reciprocating air flow for Li-ion battery thermal management to improve temperature uniformity", Journal of Power Sources, 196(13), pp. 5685-5696.

[13] Panchal, S., Khasow, R., Dincer, I., Agelin-Chaab, M., Fraser, R., and Fowler, M. (2017), "Thermal design and simulation of mini-channel cold plate for water-cooled large sized prismatic Lithium-ion battery", Applied Thermal Engineering, Vol 122, 80-90.

[14] Panchal, S., Mathewson, S., Fraser, R., Culham, R., and Fowler, M. (2015), "Thermal management of lithium-ion pouch cell with indirect liquid cooling using dual cold plates approach", SAE International Journal of Alternative Powertrains, Vol 4, 293-307.

[15] He, F., Akram AMS, A., Roosien, Y., Tao, W., Geist, B. and Singh, K. (2017), "Reduced-order Thermal Modeling of Liquid-cooled Lithium-ion Battery Pack for EVs and HEVs", Chrysler Tech Center,1000 Chrysler Dr, Auburn Hills, MI, USA.

[16] Kizilel, R., Sabbah, R., Selman, J.R., and Al-Hallaj, S. (2009), "An alternative cooling system to enhance the safety of Li-ion battery packs", Journal of Power Sources, 194(2), pp. 1105-1112.

[17] Fathabadi, H. (2014), "High thermal performance lithium-ion battery pack including hybrid active-passive thermal management system for using in hybrid/electric vehicles", Energy, 70, pp.529-538.

[18] Saw, L., Ye, Y. and Tay, A. (2013), "Electrochemical-thermal analysis of 18650 Lithium Iron Phosphate cell", Energy Conversion and Management, 75, pp.162-174.

[19] Wu, B., Yufit, V., Marinescu, M., Offer, G., Martinez-Botas, R. and Brandon, N. (2013), "Coupled thermalelectrochemical modelling of uneven heat generation in lithium-ion battery packs", Journal of Power Sources, 243, pp.544-554.

[20] Moffat, R. (1988), "Describing the uncertainties in experimental results. Experimental Thermal and Fluid Science", 1(1), pp.3-17. 